\title{
Comparison of Performance of Rapid Petrifilm Test Method and Standard Test Method for Enumeration of Aerobic Microorganisms, Coliforms and E.coli in Food
}

\author{
N.K.A. Lakmini and T. Madhujith ${ }^{1}$ \\ Postgraduate Institute of Agriculture \\ University of Peradeniya \\ Sri Lanka
}

\begin{abstract}
Assuring food safety is currently a global challenge. Frequent testing of food samples for the presence of microorganisms should be performed as part of any food safety program. The conventional tests are in general laborious and time consuming. In this context, the use of alternative rapid tests becomes important. The objective of this study was to compare the performance of Petrifilm test method and standard colony count method for enumerating aerobes, Coliforms and Escherichia coli. The Aerobic Plate Count (APC), Coliform Plate Count $(C P C)$ and E. coli obtained using Rapid Petrifilm test method were compared with the corresponding standard methods using 24 samples of 3 different foods, namely poultry, ready to serve drink (RTS) and milk powder. The counts obtained using Rapid Petrifilm method were not significantly $(p<0.05)$ different from the counts obtained using the conventional methods. The APC obtained using Rapid Petrifilm test method showed a strong correlation with the corresponding conventional method for poultry, RTS and milk powder. The correlation coefficients between the two methods for APC were $0.9934,0.9988$ and 0.9978 for poultry, RTS and milk powder, respectively. The corresponding correlation coefficients for Coliform counts were 0.9891, 0.9940 and 0.9990 while the corresponding correlation coefficients for E. coli were 0.9817, 0.9969 and 0.9983, respectively. The study shows that the Rapid Petrifilm Test method can effectively be used for $A P C$ and $C P C$ for foods.
\end{abstract}

Keywords: Aerobic plate count, coliforms, E. coli, rapid Petrifilm test

\section{INTRODUCTION}

Assuring the safety of food has been a global challenge. In this exercise, frequent testing of foods by analytical laboratories plays a significant role. Food testing laboratories are required to provide accurate analytical reports in a timely manner. Testing of food for microbiological parameters is important in determining the quality and hygiene of products. It is also helpful to allow corrective actions to be taken during the production process.

The conventional techniques used for enumeration of microorganisms in foods are laborious and material intensive as they involve a number of steps including rehydration of culture media, sterilization of media and equipment and sample preparation. Following inoculation of culture media, incubation at a given temperature is essential in most procedures.

1 Department of Food Science and Technology, Faculty of Agriculture, University of Peradeniya, Peradeniya, Sri Lanka

* Author for correspondence: madujith@yahoo.com 
Incubation is time consuming and requires continuous monitoring. Moreover, incubation of inoculated petri dishes and test tubes occupy a large amount of space in incubators. Furthermore, traditional techniques of microbiological analysis do not meet the current needs of having immediate analytical results in order to be able to make decisions in the process of food manufacturing, especially in HACCP application systems concerned with quality assurance. Therefore, rapid testing techniques for microorganisms have gained steady acceptance over the past few years. Many rapid microbiological methods have been evolved among which, Petrifilm method is considered to be one of the best known alternatives.

Petrifilms are ready-to-use products, which are composed of rehydratable films coated with standard nutrients, a cold water soluble gelling agent, and indicators that facilitate colony enumeration. Petrifilms eliminate the need for preparation of traditional media bringing many advantages over traditional enumeration techniques. Though development of modern techniques provides alternatives to existing standard methods, it is important to evaluate the effectiveness of new methods.

In this backdrop, the main objective of the present study was to evaluate the applicability of the Petrifilm method for enumerating of aerobic microorganisms, E. coli and coliforms by comparing the results of Petrifilm method with that of standard enumerating techniques. As it is not possible to identify microorganisms in food through testing, a few main pathogens causing gastrointestinal illnesses and few indicator organisms were identified and used as reference microorganisms for the study. In order to use the Petrifilm method as an alternative to traditional test methods, the level of recovery of selected types of microorganisms is important.

\section{MATERIALS AND METHODS}

\section{Materials}

Spray dried milk powder, ready to serve drink (RTS), and poultry meat were purchased from the local market. Peptone (0.1\%) (BDH, England), plate count agar (Lab-M, England), Violet Red Bile Agar (VRBA) (Lab-M, England), Eosin Methylene Blue (L-EMB) agar (Oxoid, England), Brilliant Green Lactose Bile (BGLB) broth (Oxoid, England) and Kovac's reagent (Biolife, Italy) and E. coli/coliform count plates and Petrifilms for aerobic count plates (3M, Minneapolis) were purchased. Enterobacter aerogenes, Escherichia coli and Bacillus cereus cultures were obtained from the microbiological culture collection of the Sri Lanka Standard Institution (SLSI).

\section{Sample preparation}

Samples of spray dried milk powder $(10 \mathrm{~g})$, poultry $(10 \mathrm{~g})$ and ready to serve drink (RTS) $(10 \mathrm{ml})$ were homogenized in $90 \mathrm{ml}$ of $0.1 \%$ peptone water and 10 fold dilutions were prepared as needed by transferring $1 \mathrm{ml}$ of sample into $9 \mathrm{ml}$ of $0.1 \%$ peptone waterEach food sample was inoculated with different quantities of Enterobacter aerogenes, E. coli and B. cereus cultures and suitable dilutions were enumerated in duplicates (Refai, 1979; American Public Health Association, 1992). 


\section{Standard pour plate method}

One milliliter of each of the diluted samples was placed on a petri dish and approximately 20 $\mathrm{ml}$ of melted medium was poured onto the plate. Subsequently, the plate was swirled to mix the sample equally throughout the plate and allowed to solidify. Pour plates prepared in duplicate of each dilution were inverted and incubated (American Public Health Association, 1992).

Plate Count Agar (PCA) was used for aerobic plate count and plates were incubated at $37{ }^{\circ} \mathrm{C}$ $\pm 1{ }^{\circ} \mathrm{C}$ for $72 \mathrm{~h}$ (SLS (1982b). For enumeration of coliforms and E. coli, VRBA or EMB Agar medium was used. The plates carrying coliforms were incubated at $37{ }^{\circ} \mathrm{C}$ for $72 \mathrm{~h}$ while the plates carrying E. coli were incubated at $44^{\circ} \mathrm{C}$ for 24 to $48 \mathrm{~h}$ (SLS, 1982a).

\section{Petrifilm method}

Petrifilm was placed on a flat surface and $1 \mathrm{ml}$ of sample of each dilution was placed at the centre of the bottom film by lifting the top film. The top film was carefully replaced and the sample was spread using the supplied plastic spreader. The aerobic count Petrifilms were incubated at $37^{\circ} \mathrm{C} \pm 1{ }^{\circ} \mathrm{C}$ for 24 to $48 \mathrm{~h}$ and the resulting red coloured colonies were counted and expressed as the aerobic plate count per gram or per milliliter basis. The total coliform count Petrifilms (CCP) were incubated at $37^{\circ} \mathrm{C} \pm 1^{\circ} \mathrm{C}$ for 24 to $48 \mathrm{~h}$ and the resulting blue coloured colonies developed on the film were counted and expressed on per gram or milliliter basis. The sum of blue and red colonies was expressed as total coliform counts on per gram or ml basis (Blackburn et al., 1996).

To confirm colonies of coliforms, a representative number of colonies were selected from the plates incubated at $37{ }^{\circ} \mathrm{C} \pm 1^{\circ} \mathrm{C}$, transferred into a tube containing BGLB broth and then incubated at $37{ }^{\circ} \mathrm{C} \pm 1^{\circ} \mathrm{C}$. Tubes were examined for 24 and $48 \mathrm{~h}$ for gas production. The number of coliforms per gram or milliliter was determined by multiplying the number of colonies confirmed in BGLB by the dilution factor. For confirmation of E. coli, a representative number of colonies were selected from plates incubated at $37{ }^{\circ} \mathrm{C}$ and transferred into a tube containing BGLB broth. Simultaneously, the colonies were transferred into tubes containing peptone water and the tubes were incubated at $44{ }^{\circ} \mathrm{C}$. The BLGB tubes were examined at 24 and $48 \mathrm{~h}$ for gas production and the tubes carrying peptone water were examined for Indole ring formation with Kovacs' reagent.

\section{Statistical Analysis}

Colony counts were converted to $\log 10$ values and the geometric means were determined. Regression analysis was used to compare Petrifilm aerobic plate count, total coliform count and E. coli count with the representative standard methods using Microsoft Excel Software version 2007 (De Sousa et al., 1985).

\section{RESULTS AND DISCUSSION}

Bacterial counts were converted to $\log 10$ counts per gram or milliliter by taking the dilution factor into consideration. Colony counts for both Petrifilm and standard method were obtained for 24 food samples of three foods (milk powder, RTS and poultry). Colony counts were converted to $\log 10$ values and regression analysis was performed to compare Petrifilm 
method and standard method using aerobic plate count, total coliform count and E. coli counts.

Table 1. Comparison of aerobic plate counts obtained by standard plating and Petrifilm methods

\begin{tabular}{|c|c|c|c|c|}
\hline Product & $\begin{array}{c}\text { Trial } \\
\text { no }\end{array}$ & $\frac{\text { Standard plate count }}{\text { Mean }}$ & $\frac{\text { Petrifilm count }}{\text { Mean }}$ & $\begin{array}{l}\text { Difference in mean } \\
\text { (log values) }\end{array}$ \\
\hline \multirow[t]{8}{*}{ Milk powder } & 1 & 1.658 & 1.735 & 0.078 \\
\hline & 2 & 1.892 & 1.970 & 0.078 \\
\hline & 3 & 1.549 & 1.618 & 0.069 \\
\hline & 4 & 2.579 & 2.591 & 0.012 \\
\hline & 5 & 2.301 & 2.380 & 0.079 \\
\hline & 6 & 2.863 & 2.919 & 0.056 \\
\hline & 7 & 3.412 & 3.490 & 0.078 \\
\hline & 8 & 3.053 & 3.086 & 0.033 \\
\hline \multirow[t]{8}{*}{ RTS } & 1 & 1.318 & 1.409 & 0.091 \\
\hline & 2 & 1.736 & 1.715 & -0.021 \\
\hline & 3 & 1.926 & 1.956 & 0.029 \\
\hline & 4 & 2.008 & 1.977 & -0.032 \\
\hline & 5 & 2.895 & 2.908 & 0.014 \\
\hline & 6 & 2.929 & 2.962 & 0.033 \\
\hline & 7 & 3.611 & 3.683 & 0.072 \\
\hline & 8 & 3.579 & 3.629 & 0.050 \\
\hline \multirow[t]{8}{*}{ Poultry } & 1 & 1.772 & 1.806 & 0.034 \\
\hline & 2 & 1.96 & 1.833 & -0.128 \\
\hline & 3 & 2.491 & 2.578 & -0.087 \\
\hline & 4 & 2.780 & 2.738 & -0.042 \\
\hline & 5 & 2.892 & 2.914 & 0.022 \\
\hline & 6 & 3.518 & 3.602 & 0.084 \\
\hline & 7 & 3.564 & 3.688 & 0.124 \\
\hline & 8 & 3.681 & 3.805 & 0.124 \\
\hline
\end{tabular}

Table 2. Comparison total coliform counts by standard and Petrifilm methods

\begin{tabular}{lcccc}
\hline \multirow{2}{*}{ Product } & $\begin{array}{c}\text { Trial } \\
\text { no. }\end{array}$ & Standard count & Petrifilm count & $\begin{array}{c}\text { Difference in mean } \\
\text { (log values) }\end{array}$ \\
\hline Milk powder & 1 & Mean & Mean & -0.025 \\
& 2 & 1.254 & 1.230 & -0.002 \\
& 3 & 1.371 & 1.368 & 0.016 \\
& 4 & 1.711 & 1.727 & 0.077 \\
& 5 & 1.852 & 1.929 & -0.006 \\
& 6 & 2.903 & 2.897 & 0.044 \\
RTS & 7.447 & 3.491 & 0.061 \\
& 8 & 3.518 & 3.579 & 0.010 \\
& 1 & 3.851 & 3.860 & -0.069 \\
& 2 & 1.606 & 1.537 & 0.057 \\
& 3 & 1.633 & 1.690 & 0.040 \\
& 4 & 2.000 & 2.040 & -0.090 \\
& 5 & 2.450 & 2.360 & 0.014
\end{tabular}


N.K.A. Lakmini and T. Madhujith

\begin{tabular}{ccccc} 
& 6 & 2.892 & 2.897 & 0.005 \\
\multirow{3}{*}{ Poultry } & 7 & 3.591 & 3.602 & 0.011 \\
& 8 & 3.477 & 3.380 & -0.097 \\
& 1 & 0.997 & 1.142 & 0.144 \\
& 2 & 1.184 & 1.035 & -0.149 \\
& 3 & 1.511 & 1.549 & 0.038 \\
4 & 1.598 & 1.404 & -0.193 \\
& 5 & 2.281 & 2.390 & 0.110 \\
6 & 2.591 & 2.680 & 0.089 \\
& 7 & 3.410 & 3.500 & 0.090 \\
\hline
\end{tabular}

Table 3. Comparison of E. coli counts obtained by standard and Petrifilm methods

\begin{tabular}{lcccc}
\hline Product & $\begin{array}{c}\text { Trial } \\
\text { no. }\end{array}$ & Standard count & Petrifilm count & $\begin{array}{c}\text { Difference in mean } \\
\text { (log values) }\end{array}$ \\
\cline { 2 - 4 } Milk powder & 1 & 1.228 & Mean & 0.114 \\
& 2 & 1.230 & 1.342 & 0.066 \\
& 3 & 1.724 & 1.296 & 0.078 \\
& 4 & 1.860 & 1.802 & 0.023 \\
& 5 & 2.672 & 2.644 & -0.023 \\
& 6 & 2.998 & 3.060 & 0.062 \\
RTS & 7 & 3.460 & 3.545 & 0.085 \\
& 8 & 3.757 & 3.824 & 0.067 \\
& 1 & 1.453 & 1.468 & 0.015 \\
& 2 & 1.627 & 1.694 & 0.067 \\
& 3 & 1.926 & 1.980 & 0.054 \\
& 4 & 2.272 & 2.371 & 0.099 \\
Poultry & 5 & 2.461 & 2.548 & 0.087 \\
& 6 & 2.579 & 2.555 & -0.024 \\
& 7 & 3.773 & 3.764 & -0.009 \\
& 8 & 3.591 & 3.534 & -0.057 \\
& 1 & 1.339 & 1.479 & 0.140 \\
& 2 & 1.781 & 1.912 & 0.131 \\
& 3 & 2.621 & 2.422 & -0.199 \\
& 4 & 1.793 & 1.934 & 0.141 \\
& 5 & 2.939 & 2.866 & -0.073 \\
& 6 & 3.217 & 3.419 & 0.203 \\
& 7 & 3.869 & 3.854 & -0.015 \\
& 8 & 3.936 & 3.989 & 0.052 \\
\hline
\end{tabular}

Linear least square regression of colony counts obtained through Petrifilm and standard methods showed a high linearity. The correlation coefficient (R2) between the Petrifilm APC and the standard aerobic colony count for milk powder, RTS and poultry were 0.9988 , 0.9978 and 0.9934 , respectively (Fig. 1). The correlation coefficient of total coliform count on Petrifilm CCP and the standard VRBA plates showed R2 values of 0.999, 0.994 and 0.9891 for milk powder, RTS and poultry, respectively (Fig. 2). Furthermore, comparison of E. coli counts by the two methods for milk powder, RTS and poultry showed R2 values of $0.9983,0.9969$ and 0.9817 , respectively (Fig. 3) 


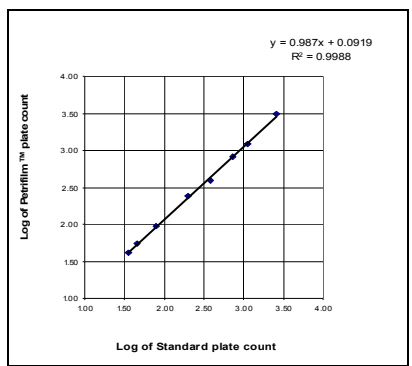

(a)

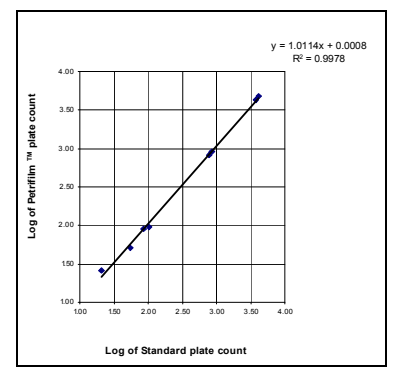

(b)

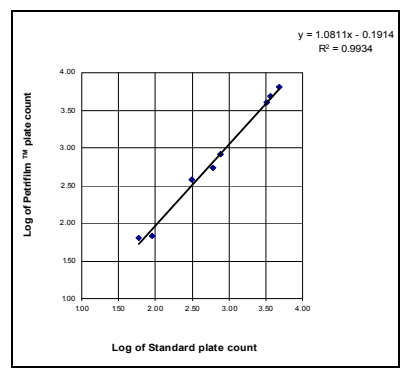

(c)

Fig. 1. Relationship between the colony counts obtained by Petrifilm aerobic plate count and conventional standard plate method for (a) milk powder, (b) RTS and (c) poultry

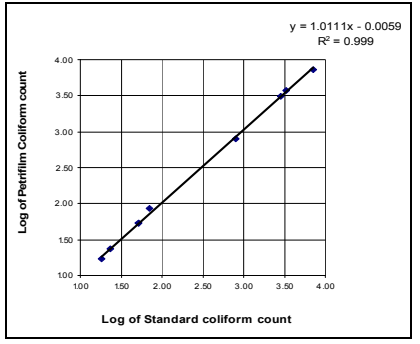

(a)

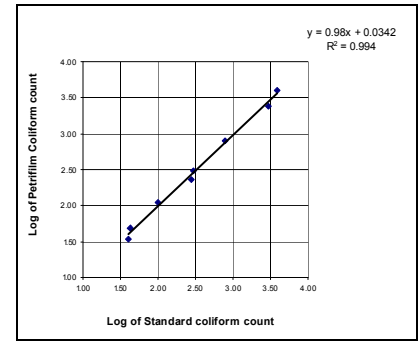

(b)

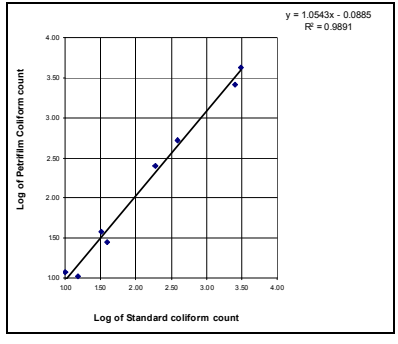

(c)

Fig. 2. Relationship between the colony counts obtained of Petrifilm coliform plate count with conventional standard plate method in (a) milk powder, (b) RTS and (c) poultry

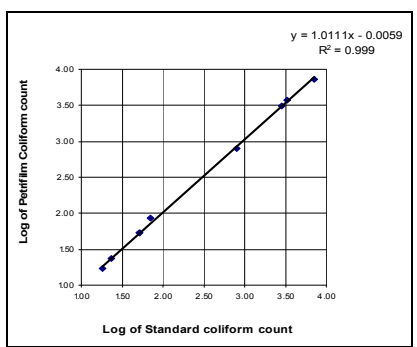

(a)

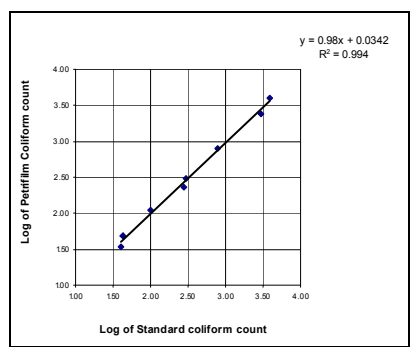

(b)

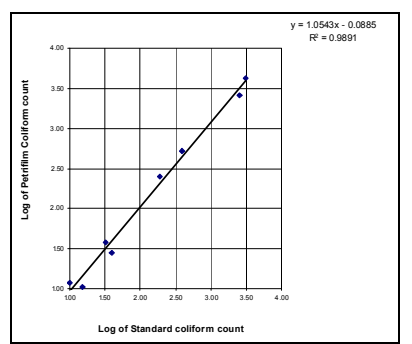

(c)

Fig. 3. Relationship between the colony counts obtained by Petrifilm E. coli count and conventional standard plate method for (a) milk powder, (b) RTS and (c) poultry

\section{CONCLUSIONS}

The colony counts of E. coli, coliform and aerobic microorganisms obtained by Petrifilm method are well correlated with the counts obtained by conventional standard plate techniques for milk powder, RTS and poultry samples. Therefore, the rapid Petrifilm technique can effectively be applied for routine laboratory analysis of regular food samples 
as a convenient alternative to conventional method for enumeration of aerobic microorganisms, coliforms and E. coli.

\section{REFERENCES}

American Public Health Association (1992). Compendium of Methods for the Microbiological Examination of Foods, 3rd Ed. APHA, Washington, DC.

Blackburn, C. de W., Baylis, C.L. and Petitt, S.B. (1996). Evaluation of Petrifilm methods for enumeration of aerobic flora and coliforms in a wide range of foods, Letters in Applied Microbiology. pp. 137-140.

De Sousa, G.B., Tamagnini, L.M., Gonzalez, R.D. and Budde, C.E. (2005). Evaluation of Petrifilm Method for enumerating aerobic bacteria in Crottin Goat's Cheese, Revista Argentina de Microbiologia 37, 214-216

Refai, M.K. (1979). Manual of Food quality control - Microbiological Analysis, Food \& Agricultural Organization, Via delle Terme di Caracalla, 00100 Rome, Italy. Vol. 4

SLS. (1982a). Microbiological Test Methods: Detection and Enumeration of Coliforms, Fecal coliforms and Escherichia coli SLS 516 part 3.

SLS. (1982b). Microbiological Test Methods: General Guidance for Enumeration of Microorganisms colony count technique at $30^{\circ} \mathrm{C}$ SLS 516 part 1 